\title{
Exosome-mediated miR-25/miR-203 as a potential biomarker for esophageal squamous cell carcinoma: improving early diagnosis and revealing malignancy
}

\author{
Zhun Liu ${ }^{1 \#}$, Ying Huang ${ }^{2 \#}$, Ziyang Han ${ }^{1}$, Zhimin Shen ${ }^{1}$, Shaobin Yu ${ }^{1}$, Tao Wang ${ }^{3}$, Zhaonan Dong ${ }^{3}$, \\ Mingqiang Kang ${ }^{1,4}$ \\ ${ }^{1}$ Department of Thoracic Surgery, Fujian Medical University Union Hospital, Fuzhou, China; ${ }^{2}$ Department of Infusion, Fujian Medical University \\ Union Hospital, Fuzhou, China; ${ }^{3}$ Jiangsu Engineering Research Center for Tumor Molecular Diagnosis, Suzhou, China; ${ }^{4}$ Key Laboratory of \\ Gastrointestinal Cancer (Fujian Medical University), Ministry of Education, Fuzhou, China \\ Contributions: (I) Conception and design: Z Liu, Y Huang, Z Han, M Kang; (II) Administrative support: Z Han; (III) Provision of study materials or \\ patients: Z Liu, Z Shen, S Yu; (IV) Collection and assembly of data: Z Liu, Y Huang, Z Han; (V) Data analysis and interpretation: T Wang, Z Dong, \\ M Kang; (VI) Manuscript writing: All authors; (VII) Final approval of manuscript: All authors. \\ "These authors contributed equally to this work. \\ Correspondence to: Ziyang Han, MM; Mingqiang Kang, MD. Department of Thoracic Surgery, Fujian Medical University Union Hospital, No. 29, \\ Xinquan Rd, Gulou District, Fuzhou 350001, China. Email: ziyang_h9@163.com; kangmq_kmq@163.com.
}

Background: Esophageal squamous cell carcinoma (ESCC) is the leading cause of cancer death in men and women worldwide. The poor prognosis and rapid increase in ESCC incidence highlight the need to promote early detection and prediction. Identifying key molecular targets involved in ESCC monitoring and progression is critical for ESCC patients.

Methods: This study examined miR-25/miR-203 as a biomarker for ESCC patients. Real-time quantitative polymerase chain reaction (PCR) was used to detect miR-25/miR-203 expression levels in tissues and serum exosomes, and MiR-25/miR-203 upregulation was confirmed in ESCC.

Results: We found that the miR-25/miR-203 ratio in cancer tissues from 36 ESCC patients was significantly enhanced compared with that in adjacent tissues. Moreover, the serum level of miR-25/miR203 in 57 ESCC patients was higher than that in 31 healthy volunteers. Intriguingly, in 38 ESCC patients, the level of miR-25/miR-203 decreased significantly after surgery. Using ROC curve statistical analysis, we found that each group of miR-25/miR-203 had obvious sensitivity and high specificity. The miR-25/miR203 relationship with the clinicopathological features of ESCC patients was also analyzed. MiR-25/miR203 was significantly associated with the ESCC TNM-stage and lymph node metastasis, which predicts the prognosis of ESCC and reflects tumor progression.

Conclusions: This study highlights the feasibility of using exosome-mediated miR-25/miR-203 as a vital noninvasive biomarker for the detection and treatment monitoring of ESCC.

Keywords: Biomarkers; microRNAs; exosomes; serum; esophageal squamous cell carcinoma (ESCC)

Submitted Jun 29, 2021. Accepted for publication Oct 24, 2021.

doi: $10.21037 /$ tcr-21-1123

View this article at: https://dx.doi.org/10.21037/tcr-21-1123

\section{Introduction}

Esophageal squamous cell carcinoma (ESCC) is one of the most common malignancies worldwide (1), and its incidence has increased six-fold over the past four decades
$(2,3)$. Despite the rapid development of conventional surgical resection, chemotherapy, radiotherapy, and other esophageal cancer treatment strategies, the 5 -year survival rate is still less than $20 \%(4,5)$. Therefore, it is imperative to 
identify a novel noninvasive biomarker for ESCC diagnosis and treatment monitoring with high stability and specificity.

The exosome is a nanosized vesicular body secreted by cells, which encloses biologically active substances and participates in many important physiological and pathological processes $(6,7)$. The exosomal membrane protects the exosome contents from degradation by enzymes in the extracellular environment (8). MicroRNAs are a class of noncoding small ribonucleic acids (RNAs) that are ubiquitous in organisms. Growing evidence has indicated that they have important regulatory effects on a variety of biological activities, such as growth, development, cell proliferation, differentiation, and metabolism (9-11). Numerous studies have demonstrated that the dysregulated expression of several key miRNAs is closely associated with cancer malignancies as well as the prognosis and prediction of response to treatment in different human cancers (12-14). MiR-203 has been shown to be abnormally expressed in different cancers and to promote abnormal cancer proliferation, invasion, and metastasis $(15,16)$. MiR-203 is also hypermethylated in esophageal cancer. Hypermethylation is an important modification that causes the expression of miR-203 to decrease, which promotes the development and progression of ESCC. Furthermore, when miR-203 is upregulated over 4.5 -fold, it is significantly associated with lymph node metastasis (17). MiR-25 belongs to the miR-106b-25 cluster, which is related to hepatocellular tumors (18). Kim et al. (19) also found that, in humans, miR-25 is significantly upregulated in gastric cancer tissues and adjacent tissues compared with normal tissues. The high expression of miR25 may be a high-risk-related factor for gastric cancer tissue penetrating the serosa, lymph nodes, and distant metastasis. It is known that microRNAs are stable in body fluids and are not susceptible to environmental factors (16). Considering the desire for a novel, noninvasive, convenient, clinical tool, serum miR-25/miR-203 holds great potential for future application as a new biomarker for ESCC.

The present study aimed to characterize miR-25/miR-203 in peripheral serum, with a view to developing the diagnostics and monitoring of ESCC. We present the following article in accordance with the STARD reporting checklist (available at https://dx.doi.org/10.21037/tcr-21-1123).

\section{Methods}

\section{Patients}

In 2017, ESCC patients undergoing thoracoscopic surgery combined with laparoscopic surgery at the Department of Thoracic Surgery, Fujian Medical University Union Hospital were selected for this study. Surgical specimens were collected straight away post-operation from each patient, by using scalpels to cut off a sample of the cancer tissues as well as adjacent tissues. All of the specimens were immediately stored in liquid nitrogen. We collected preoperative peripheral blood from 57 ESCC patients and postoperative peripheral blood from 38 ESCC patients and used peripheral blood from 31 healthy volunteers as a control. Clinical staging was determined according to the American Joint Committee on Cancer (AJCC) 8th edition of ESCC TNM-Staging. The study was approved by the Ethics Committee of Fujian Medical University Union Hospital. Written informed consent was obtained from all participants. The registration number is 2020KY099. The study is in accordance with the Helsinki Declaration (as revised in 2013).

\section{Serum exosome isolation}

Serum exosomes were isolated using the modified polyethylene glycol (PEG) separation method. An appropriate amount of polyethylene glycol 6000 (average molecular mass 6000) was dissolved in pure water and sodium chloride was added to make sodium chloride solution $1 \mathrm{M}$, thereby obtaining a storage solution of a $2 \times$ concentration. The serum was then mixed with one volume of the above PEG solution and incubated at $4{ }^{\circ} \mathrm{C}$ overnight. It was then spun in a refrigerated centrifuge at the highest speed for one hour, and the pellet was collected and resuspended in PBS.

\section{RNA extraction and reverse transcription}

Total RNA extraction with TRIzol was performed as follows: (I) the homogenized sample was placed at room temperature for $5 \mathrm{~min}$, and 0.2 times the volume of chloroform was added. The samples were then spun for $15 \mathrm{~s}$, mixed well, placed at $15-30^{\circ} \mathrm{C}$ for $2-3 \mathrm{~min}$, and then centrifuged at $4{ }^{\circ} \mathrm{C}$ and $13,000 \mathrm{rpm}$. Centrifugation resulted in a lower red phenol-chloroform phase, an intermediate phase, and an upper colorless aqueous phase. (II) The upper aqueous phase was carefully transferred to a new centrifuge tube, one volume of isopropanol was added, and the mixture was gently inverted. After incubation at room temperature for $10 \mathrm{~min}$, the sample was centrifuged at 13,000 rpm for 15 min at $4{ }^{\circ} \mathrm{C}$, and the supernatant was discarded. (III) The 
pellet was mixed with $70 \%$ alcohol and inverted several times to wash off the salt contained in the precipitate. If the precipitate is larger, the pellet was broken up. The sample was then centrifuged again at 13,000 rpm for $10 \mathrm{~min}$ at $4{ }^{\circ} \mathrm{C}$, the supernatant was discarded, and the procedure was repeated one more time. (IV) The sample was placed on the ultraclean workbench, and the tube cover was opened, allowing the pellet to dry at room temperature, and then the appropriate amount of RNase-free water was added to dissolve and mix up the pellet, and this was immediately followed by centrifugation. (V) A total of $1.2 \mu \mathrm{L}$ was used to analyze the RNA concentration and purity. Reverse transcription was performed as follows. (I) Two microliters of RNA template was pre-denatured at $70^{\circ} \mathrm{C}$ for $10 \mathrm{~min}$. (II) The sample was incubated in an ice bath for $5 \mathrm{~min}$. (III) The transcription system was prepared in the following order: $25 \mathrm{mmol} / \mathrm{L} \mathrm{MgCl}_{2}(4 \mu \mathrm{L}) \rightarrow$ reverse transcription buffer $(2 \mu \mathrm{L}) \rightarrow 10 \mathrm{mmol} / \mathrm{L}$ dNTP $(2 \mu \mathrm{L}) \rightarrow$ RNase $(0.5 \mu \mathrm{L}) \rightarrow$ template $(2 \mu \mathrm{L}) \rightarrow$ random primer or specific downstream primer $(1 \mu \mathrm{L}) \rightarrow$ DEPC water $(8.5 \mu \mathrm{L})$. (IV) The mixture was incubated for $15 \mathrm{~min}$ at room temperature. (V) Finally, the mixture was incubated at $42{ }^{\circ} \mathrm{C}$ for $1 \mathrm{~h}$.

\section{Quantitative real-time PCR}

The Mx3000P QPCR system (Agilent Technologies, Santa Clara, CA, USA) was used to carry out qRT-PCR with the SYBR PremixExX Taq kit (Takara, Shiga, Japan). Primer sequences for qRT-PCR were obtained from the online database miRbase (http://www.miRbase.org/). Table 1 lists the primer sequences. The $\Delta \Delta \mathrm{Ct}$ was calculated by subtracting the average $\mathrm{Ct}$ value of the corresponding reference gene from the average Ct value of the miRNAs of interest. The $\Delta \mathrm{Ct}$ was then calculated by subtracting the $\Delta \mathrm{Ct}$ of precancerous tissue or the average expression in healthy volunteers from the $\Delta$ Ct of ESCC patients. The fold change in gene expression was calculated with the equation $2^{-\Delta \Delta \mathrm{Ct}}$. The expression levels of miRNAs were converted into dichotomous variables by splitting the samples into two classes (high and low expression), using the respective mean level expression of miRNA as a cut-off point.

\section{Data statistics}

All statistical analyses were performed using SPSS 19.0 (SPSS Inc., Chicago, USA) using the mean \pm standard deviation. $\mathrm{P}<0.05$ was considered statistically significant. The two groups were compared with the use of one-way analysis of variance; ROC curves and the area under the ROC curve (AUC) were used to assess the specificity and sensitivity of each miRNA for the diagnosis of ESCC. The rank-sum test was used to compare differences in serum miR-25 and miR203 expression between ESCC patients and healthy controls. The chi-square test was used to determine the relationship between the expression of these serum miRNAs and the clinical-pathological parameters of ESCC.

\section{Results}

The up-regulation of serum exosome miR-25/miR-203 in primary ESCC

To verify the expression of miR-25/miR-203 in the ESCC serum samples, we analyzed the levels of miR-25/miR-203 in the serum of 57 ESCC patients and 31 healthy volunteers. We found that the miR-25/miR-203 ratio in ESCC was significantly higher than that in the control group, as shown by the ROC curve (AUC $=0.821$, sensitivity $=71.9 \%$, specificity $=96.6 \%$, Figure $1 \mathrm{~A}, 1 \mathrm{~B})$. We also collected 36 ESCC tumors and adjacent tissues and confirmed that the expression level of miR-25/miR-203 in tumor tissues was higher than that in adjacent tissues, as shown by the ROC curve (AUC $=0.924$, sensitivity $=91.7 \%$, specificity $=80.6 \%$ ), as shown in Figure 1C,1D. To determine whether miR25/miR-203 has value in monitoring the effect of ESCC tumor resection and recurrence. we analyzed the expression levels of serum miR-25/miR-203 in the preoperative and postoperative serum of $38 \mathrm{ESCC}$ patients and showed that the expression level of $\mathrm{miR}-25 / \mathrm{miR}-203$ was significantly reduced after surgery, as indicated by the ROC curve (AUC $=0.831$, sensitivity $=97.4 \%$, specificity $=65.8 \%$, as shown in Figure $1 E, 1 F$.

\section{The clinical significance of miR-25/miR-203 in patients with primary ESCC}

To confirm the clinical significance of serum miR-25/miR203 upregulation in ESCC, we analyzed the clinical data of 57 cases of ESCC (Table 1). Pearson's $\chi^{2}$ analysis of miR25/miR-203 expression and clinicopathological features indicated that miR-25/miR-203 upregulation was associated with ESCC clinical stages. Our analysis showed that miR25/miR-203 overexpression was associated with $\mathrm{T}$ stage $(\mathrm{P}=0.043)$ and $T N M$ stage $(\mathrm{P}=0.013)$ but was not related to age, gender, location, smoking, tumor differentiation or hemangioma. It would therefore appear that miR-25/ 
Table 1 Clinicopathological characteristics of 57 ESCC patients with respect to miR-25/miR-203 expression

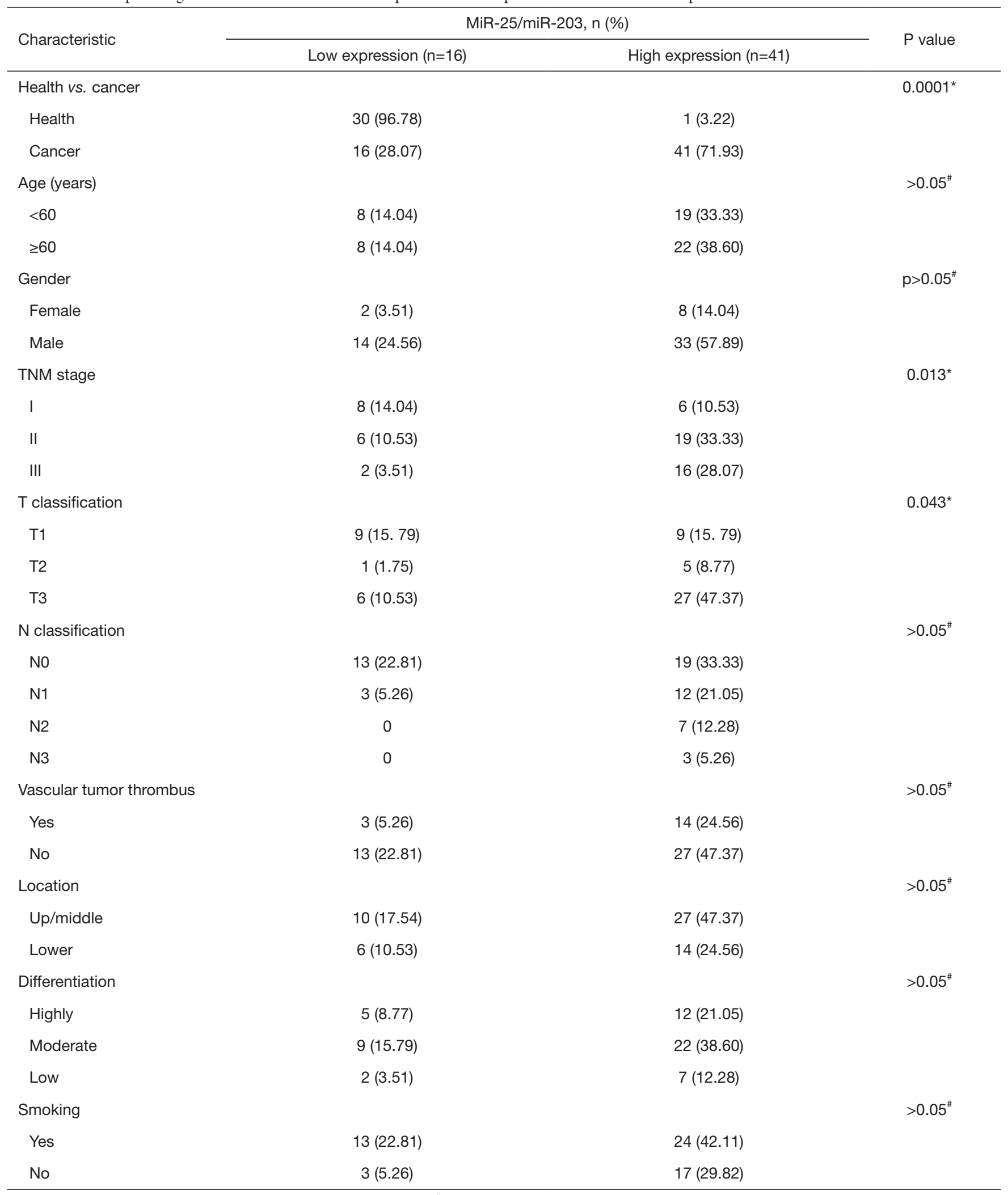

*, P value was determined using Pearson's chi-square test; ${ }^{*}$, no statistical. 

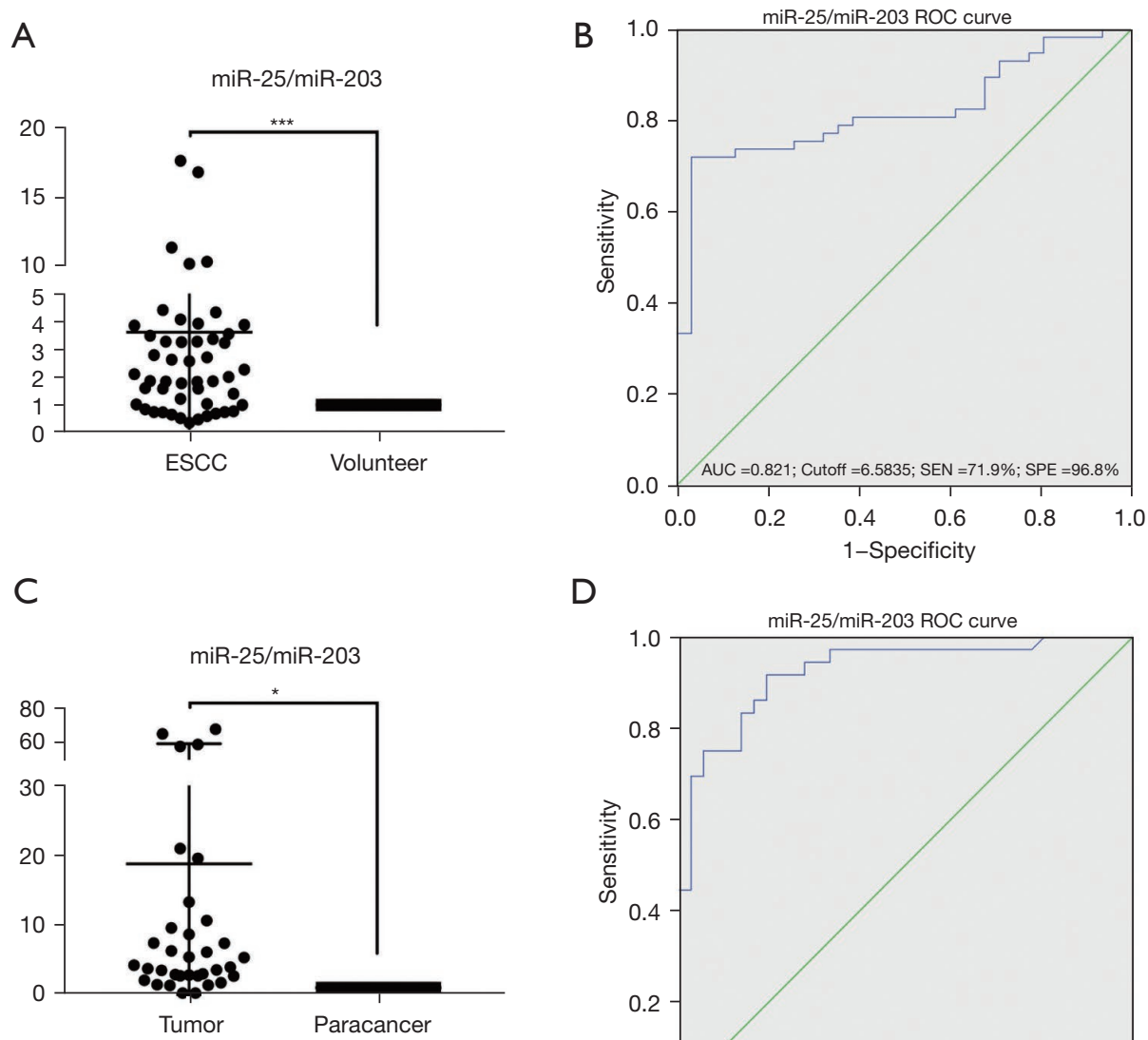

D
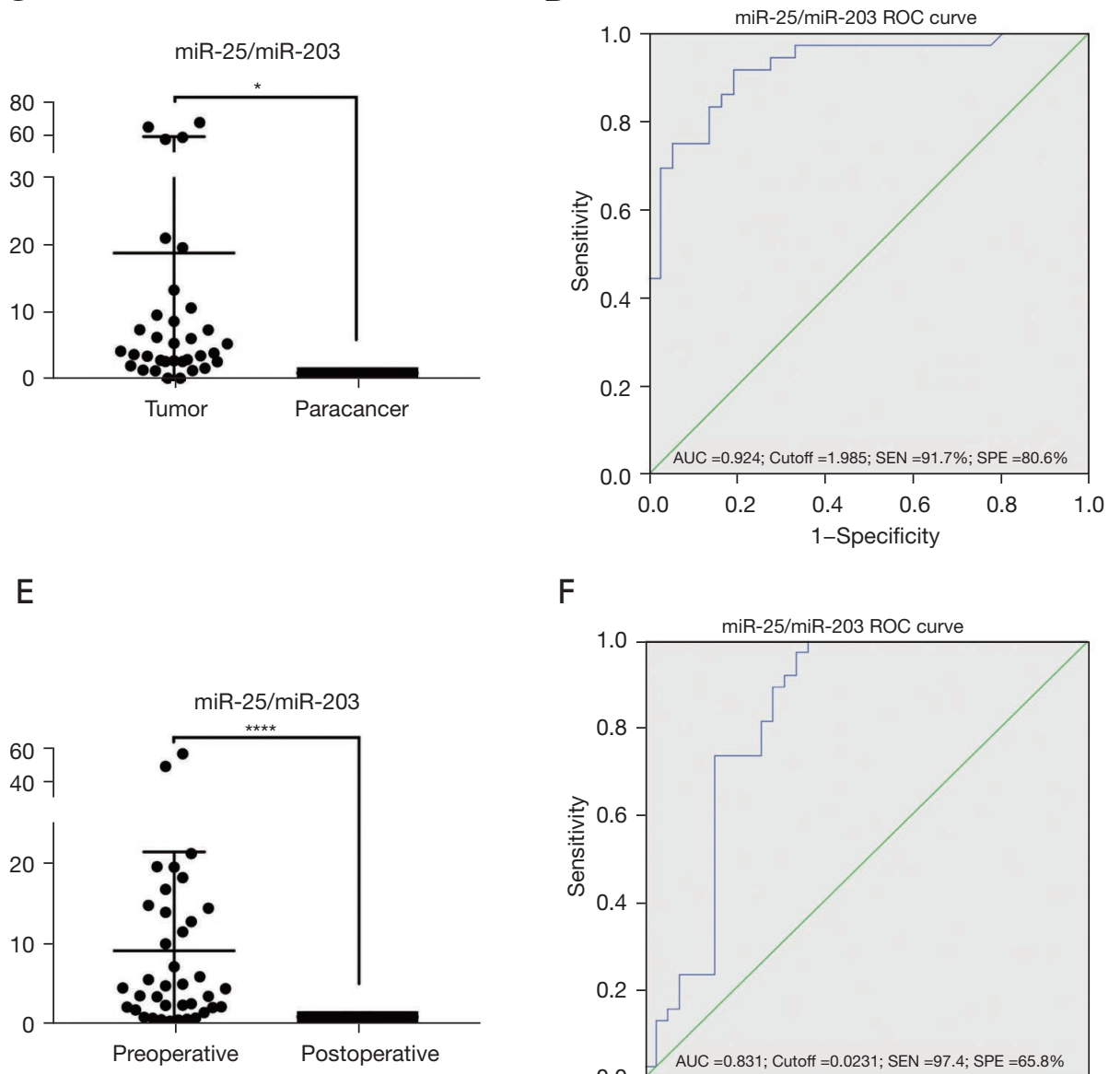

F

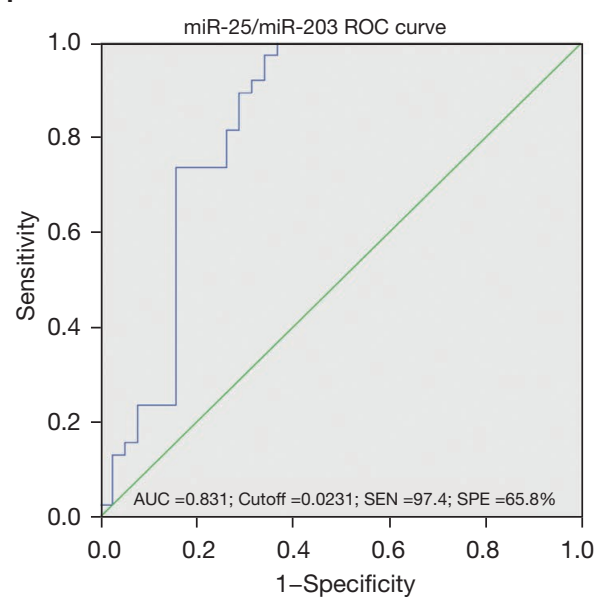

Figure 1 The up-regulation of exosome miR-25/miR-203 in primary ESCC. (A,B) The level of miR-25/miR-203 in serum was higher than that in the healthy volunteer group $(\mathrm{P}<0.05)$ : ROC curve (AUC $=0.821$, sensitivity $=71.8 \%$, specificity $=96.6 \%)$; $(\mathrm{C}, \mathrm{D})$ we also found the level of miR-25/miR-203 in tumor was higher than that in non-tumors tissue tissues $(\mathrm{P}<0.05)$ : ROC curve $(\mathrm{AUC}=0.924$, sensitivity $=91.7 \%$, specificity $=80.6 \%)$; intriguingly, $(\mathrm{E}, \mathrm{F})$ the level of miR-25/miR-203 decreased significantly after surgery $(\mathrm{P}<0.05)$ : ROC curve $(\mathrm{AUC}$ $=0.831$, sensitivity $=97.4 \%$, specificity $=65.8 \%)$. ${ }^{*} \mathrm{P}<0.05,{ }^{* * *} \mathrm{P}<0.001$, ${ }^{* * * *} \mathrm{P}<0.0001$. 
miR-203 could be used as a potential biomarker test for the diagnosis and treatment of ESCC.

\section{Discussion}

In recent decades, although therapeutic strategies and biological targets for ESCC have made great progress, the prognosis of this malignant cancer is still poor. Therefore, researchers are increasingly shifting their focus to the relationship between miRNAs and cancers. It is critical to identify specific and sensitive biomarkers for early detection, prognosis and treatment response and as potential targets for prevention and therapy (20).

The pathogenesis of ESCC is complex, and miRNAs may affect its occurrence and progression through various pathways. Studies have found that microRNA expression is significantly different between ESCC and paracancerous tissue. For example, microRNA-10a inhibits cell proliferation and metastasis of ESCC by targeting Tiam1, suggesting that microRNAs play an important role in ESCC progression (21). Research aimed at the characterization of circulating miRNA complexes in human serum revealed that circulating miRNAs not only copurified with the Ago2 ribonucleoprotein complex but also cofractionated with vesicles such as exosomes (22). Furthermore, the biological properties of the exosomal membrane allows microRNAs in exosomes to resist enzymatic degradation in the extracellular environment (8). Therefore, an exosomal miRNA with dysregulated expression was also detected in peripheral circulation, and it can be used as a biomarker for ESCC diagnosis (23).

In this study, our results were consistent with the results of previous microRNA chips showing that miR-203 was upregulated, and miR-25 was downregulated, in ESCC. MiR-25 and miR-203, which are important members of the microRNA family, are specifically expressed in epithelial tissues, and their expression levels are significantly different in cancer. They can also be used as oncogenes, and, as cancer suppressor genes, they interact with a variety of microRNA target genes to participate in different stages of cancer development. The results showed that miR-203 and miR-25 may have certain diagnostic value in ESCC, and the combination of the two may have better diagnostic performance.

The previous studies have investigated the role of miR203 in cancer (17,24-29). Cui et al. (17) found that miR203 has good diagnostic and prognostic value in patients at the early stage of ESCC. Guo et al. in vitro studies showed that knocking out microRNA-203 reduces the cisplatin chemosensitivity of osteosarcoma cell by targeting RUNX2 (26). miR-203 induces independent growth of MET characteristics and growth factors by inhibiting SNAI2 in prostate cancer (27). In addition, miR-203 also inhibits the growth of bladder cancer cells by targeting the Twist family bHLH transcription factor-1 (28). MiR203 regulates the proliferation and apoptosis of ovarian cancer cells by targeting the expression of SOCS3, and affects proliferation through the JAK-STAT pathway (29). We found that serum miR-203 was significantly higher in ESCC patients with lymph node metastasis than those without lymph node metastasis. Moreover, the serum miR-203 level was significantly lower than that before surgery. We further determined that there was a significant association between preoperative serum miR-203 levels and the pathological stage of ESCC. Guo et al. (30) showed that miR-25 was upregulated in ESCC tissues. Komatsu et al. (31) showed that serum miR-25 diagnosed ESCC with high sensitivity and specificity, which is consistent with our results, and its carcinogenesis was associated with the negative regulation of tumor suppressor genes. Preoperative patients and patients with recurrent squamous cell carcinoma also showed high miR-25 expression, and the concentration in plasma of patients after surgery was significantly reduced. These observations indicate that plasma miR-25 is beneficial for the early diagnosis of ESCC and the dynamic monitoring of recurrence of ESCC patients. When multiple serum miRNAs are combined, there is higher sensitivity and specificity for cancer diagnosis (32).

At least from a clinical practice perspective, the strength of our current research is the use of serum rather than tissue biomarkers. The extraction of serum test biomarkers is a more feasible noninvasive method that can be used for ESCC diagnostics, real-time monitoring, and assessment of treatment outcomes. The heterogeneity of individual cancers is an important issue in the study of biomarkers using ESCC tissue, which may affect the reproducibility of research results and, thus, affect the transformation of reagents into clinical practice (33). Using blood or serumderived biomarkers are considered strategies to overcome this particular problem. After proper validation, such biomarkers may be more robust and, therefore, easier to translate into clinically useful blood tests. Furthermore, the two selected miRNAs with a similar dysregulation status may also exist in other tumors $(12-15,18)$. Thus, if the selected miR-25/miR-203 combination is a regulatory 
miRNA that controls the driver genes of ESCC, then studies of these miRNA combinations can improve the discriminability of ESCC with other cancer types $(34,35)$. However, focusing on discoveries of a single miRNA able to target multiple driver genes could be a barrier to research progress. The results of this study are similar and suggest that using a single miRNA for the diagnosis of esophageal cancer can be limiting, and the combined detection of multiple miRNAs can significantly improve diagnostic efficiency.

Inevitably, there were limitations to this study. First, because the collection of specimens occurred in the past year or two, long-term follow-up data are not available yet. Therefore, there are no complete survival data, which makes it impossible to assess the prognostic value of miR-25/miR-203 in ESCC. Moreover, our study only investigated ESCC, and no other cancer types, such as adenocarcinoma. Although it seems reasonable to expect that miR-25/miR-203 may play a role in ESCC diagnostics, real-time monitoring, and assessment of treatment outcomes, this possibility still needs investigation. However, the results of this study demonstrate significant potential for the development of exosome-mediated $\mathrm{miR}-25 / \mathrm{miR}$ 203 for ESCC diagnosis and therapeutic evaluation, and so doing more work to confirm this would be justifiable.

\section{Conclusions}

In this study, the expression level of miR-25/miR-203 in ESCC tissues and blood samples was detected. The results showed that the target microRNA was differentially expressed in ESCC and showed a significant change trend after surgery. However, there are still some shortcomings in our study, the number of cases included in our study is very limited, and a complete prognosis is not yet available. But the clinical significance of $\mathrm{miR}-25 / \mathrm{miR}-203$ in ESCC deserves further study. Taken together, through the integration of miR-25/miR-203, our results strongly demonstrated that exosome-mediated miR-25/miR-203 levels may have a better potential value in cancer diagnosis and monitoring in ESCC patients.

\section{Acknowledgments}

Funding: This study was supported by Natural Science Foundation of Fujian (2018J01294) and Startup Fund for scientific research at Fujian Medical University (Grant Number: 2019QH2023).

\section{Footnote}

Reporting Checklist: The authors have completed the STARD reporting checklist. Available at https://dx.doi. org/10.21037/tcr-21-1123

Data Sharing Statement: Available at https://dx.doi. org/10.21037/tcr-21-1123

Conflicts of Interest: All authors have completed the ICMJE uniform disclosure form (available at https://dx.doi. org/10.21037/tcr-21-1123). The authors have no conflicts of interest to declare.

Ethical Statement: The authors are accountable for all aspects of the work in ensuring that questions related to the accuracy or integrity of any part of the work are appropriately investigated and resolved. The study was approved by the Ethics Committee of Fujian Medical University Union Hospital. Written informed consent was obtained from all participants. The registration number is (2020KY099). The study is in accordance with the Helsinki Declaration (as revised in 2013).

Open Access Statement: This is an Open Access article distributed in accordance with the Creative Commons Attribution-NonCommercial-NoDerivs 4.0 International License (CC BY-NC-ND 4.0), which permits the noncommercial replication and distribution of the article with the strict proviso that no changes or edits are made and the original work is properly cited (including links to both the formal publication through the relevant DOI and the license). See: https://creativecommons.org/licenses/by-nc-nd/4.0/.

\section{References}

1. Runowicz CD, Leach CR, Henry NL, et al. American Cancer Society/American Society of Clinical Oncology Breast Cancer Survivorship Care Guideline. J Clin Oncol 2016;34:611-35.

2. Dubecz A, Solymosi N, Stadlhuber RJ, et al. Does the Incidence of Adenocarcinoma of the Esophagus and Gastric Cardia Continue to Rise in the Twenty-First Century?-a SEER Database Analysis. J Gastrointest Surg 2013;18:124-9.

3. Stavrou EP, McElroy HJ, Baker DF, et al. Adenocarcinoma of the oesophagus: incidence and survival rates in New South Wales, 1972-2005. Med J Aust 2009;191:310-4. 
4. Pennathur A, Gibson MK, Jobe BA, et al. Oesophageal carcinoma. Lancet 2013;381:400-12.

5. Syed N, Barbhuiya MA, Pinto SM, et al. Phosphotyrosine profiling identifies ephrin receptor $\mathrm{A} 2$ as a potential therapeutic target in esophageal squamous-cell carcinoma. Proteomics 2015;15:374-82.

6. Pegtel DM, Gould SJ. Exosomes. Annu Rev Biochem 2019;88:487-514.

7. Harding CV, Heuser JE, Stahl PD. Exosomes: looking back three decades and into the future. J Cell Biol 2013;200:367-71. Erratum in: J Cell Biol 2013;201:485.

8. Liu R, Gu J, Jiang P, et al. DNMT1-microRNA126 epigenetic circuit contributes to esophageal squamous cell carcinoma growth via ADAM9-EGFR-AKT signaling. Clin Cancer Res 2015;21:854-63.

9. Rosenfeld N, Aharonov R, Meiri E, et al. MicroRNAs accurately identify cancer tissue origin. Nat Biotechnol 2008;26:462-9.

10. Bartel DP. MicroRNAs: genomics, biogenesis, mechanism, and function. Cell 2004;116:281-97.

11. Ambros V. The functions of animal microRNAs. Nature 2004;431:350-5

12. Volinia S, Nuovo G, Drusco A, et al. Pluripotent stem cell miRNAs and metastasis in invasive breast cancer. J Natl Cancer Inst 2014;106:dju324. Erratum in: J Natl Cancer Inst. 2014;106:dju373.

13. Zhang X, Sai B, Wang F, et al. Hypoxic BMSC-derived exosomal miRNAs promote metastasis of lung cancer cells via STAT3-induced EMT. Mol Cancer 2019;18:40.

14. De Robertis M, Poeta ML, Signori E, et al. Current understanding and clinical utility of miRNAs regulation of colon cancer stem cells. Semin Cancer Biol 2018;53:232-47.

15. Zhu X, Er K, Mao C, et al. miR-203 suppresses tumor growth and angiogenesis by targeting VEGFA in cervical cancer. Cell Physiol Biochem 2013;32:64-73.

16. Zhao S, Han J, Zheng L, et al. MicroRNA-203 Regulates Growth and Metastasis of Breast Cancer. Cell Physiol Biochem 2015;37:35-42.

17. Cui X, Chen X, Wang W, et al. Epigenetic silencing of miR-203 in Kazakh patients with esophageal squamous cell carcinoma by MassARRAY spectrometry. Epigenetics 2017;12:698-707.

18. Li Y, Tan W, Neo TW, et al. Role of the miR-106b-25 microRNA cluster in hepatocellular carcinoma. Cancer Sci 2009;100:1234-42.

19. Kim YK, Yu J, Han TS, et al. Functional links between clustered microRNAs: suppression of cell-cycle inhibitors by microRNA clusters in gastric cancer. Nucleic Acids Res
2009;37:1672-81.

20. Gu J, Wang Y, Wu X. MicroRNA in the pathogenesis and prognosis of esophageal cancer. Curr Pharm Des 2013;19:1292-300.

21. Liu Y, Wang X, Jiang X, et al. Tumor-suppressive microRNA-10a inhibits cell proliferation and metastasis by targeting Tiam1 in esophageal squamous cell carcinoma. J Cell Biochem 2019;120:7845-57.

22. Arroyo JD, Chevillet JR, Kroh EM, et al. Argonaute2 complexes carry a population of circulating microRNAs independent of vesicles in human plasma. Proc Natl Acad Sci U S A 2011;108:5003-8.

23. Takeshita N, Hoshino I, Mori M, et al. Serum microRNA expression profile: miR-1246 as a novel diagnostic and prognostic biomarker for oesophageal squamous cell carcinoma. Br J Cancer 2013;108:644-52.

24. Lohcharoenkal $W$, Harada $M$, Lovén J, et al. MicroRNA-203 Inversely Correlates with Differentiation Grade, Targets c-MYC, and Functions as a Tumor Suppressor in cSCC. J Invest Dermatol 2016;136:2485-94.

25. Yu X, Jiang X, Li H, et al. miR-203 inhibits the proliferation and self-renewal of esophageal cancer stemlike cells by suppressing stem renewal factor Bmi-1. Stem Cells Dev 2014;23:576-85.

26. Huang Z, Huang L, Liu L, et al. Knockdown of microRNA-203 reduces cisplatin chemo-sensitivity to osteosarcoma cell lines MG63 and U2OS in vitro by targeting RUNX2. J Chemother 2021;33:328-41.

27. Qu Y, Li WC, Hellem MR, et al. MiR-182 and miR203 induce mesenchymal to epithelial transition and selfsufficiency of growth signals via repressing SNAI2 in prostate cells. Int J Cancer 2013;133:544-55.

28. Shen J, Zhang J, Xiao M, et al. miR-203 Suppresses Bladder Cancer Cell Growth and Targets Twist1. Oncol Res 2018;26:1155-65.

29. Liu HP, Zhang Y, Liu ZT, et al. MiR-203 regulates proliferation and apoptosis of ovarian cancer cells by targeting SOCS3. Eur Rev Med Pharmacol Sci 2019;23:9286-94.

30. Guo Y, Chen Z, Zhang L, et al. Distinctive microRNA profiles relating to patient survival in esophageal squamous cell carcinoma. Cancer Res 2008;68:26-33.

31. Komatsu S, Ichikawa D, Hirajima S, et al. Plasma microRNA profiles: identification of miR-25 as a novel diagnostic and monitoring biomarker in oesophageal squamous cell carcinoma. Br J Cancer 2014;111:1614-24.

32. Xie SD, Xu CY, Shen JG, et al. HER 2/neu protein expression in gastric cancer is associated with poor survival. 
Mol Med Rep 2015;12:4794.

33. Chiam K, Wang T, Watson DI, et al. Circulating Serum Exosomal miRNAs As Potential Biomarkers for Esophageal Adenocarcinoma. J Gastrointest Surg 2015;19:1208-15.

34. Agrawal N, Jiao Y, Bettegowda C, et al. Comparative

Cite this article as: Liu Z, Huang Y, Han Z, Shen Z, Yu S, Wang T, Dong Z, Kang M. Exosome-mediated miR-25/ miR-203 as a potential biomarker for esophageal squamous cell carcinoma: improving early diagnosis and revealing malignancy. Transl Cancer Res 2021;10(12):5174-5182. doi: $10.21037 /$ tcr-21-1123 genomic analysis of esophageal adenocarcinoma and squamous cell carcinoma. Cancer Discov 2012;2:899-905.

35. Volinia S, Calin GA, Liu CG, et al. A microRNA expression signature of human solid tumors defines cancer gene targets. Proc Natl Acad Sci U S A 2006;103:2257-61. 